\title{
Os riscos e danos nas intoxicações por paraquat em animais domésticos
}

\author{
The risks and injuries in the paraquat intoxication in domestic animals
}

\author{
Gisane Lanes de Almeida' Gabriela Cristina Schmitt ${ }^{\mathrm{II}}$ André Valle de Bairros ${ }^{\mathrm{III}}$ \\ Tatiana Emanuelliv ${ }^{\mathrm{V}}$ Solange Cristina Garciav
}

\section{- REVISÃO BIBLIOGRÁFICA -}

\section{RESUMO}

O paraquat é um herbicida de contato não-seletivo. É amplamente utilizado na agricultura em mais de 100 países, pois apresenta baixo custo, grande eficácia e não possui efeitos poluentes cumulativos para o solo. Porém, ele é um produto muito tóxico para humanos e animais, podendo causar intoxicações fatais, principalmente pela falta de um antídoto eficaz na reversão do quadro clínico. O paraquat atua mediante mecanismos de indução do estresse oxidativo, produção aumentada de radicais livres associada à depleção dos sistemas antioxidantes do organismo. Sua toxicidade acomete rins, fígado, músculos, cérebro, dentre outros. Os pulmões são considerados os órgãos-alvo deste herbicida, levando a severas injúrias como edema, hemorragia, inflamação intersticial e fibrose pulmonar. A falência respiratória grave é a causa comum de morte. O tratamento da intoxicação, atualmente, é baseado em medidas que diminuam a absorção e aumentem a excreção. Entretanto, o uso de agentes antioxidantes $e$ antifibróticos vem sendo estudado, pois há interesse crescente no estudo de substâncias que possam servir como antídoto nas intoxicações, uma vez que o paraquat aumenta os índices de morbidade e mortalidade.

Palavras-chave: Paraquat, herbicida, intoxicação, dano pulmonar, quadro clínico, terapêutica.

\section{ABSTRACT}

Paraquat is a nonselective contact herbicide. It is widely used in agriculture because it is inexpensive and highly

\begin{abstract}
efficient. Moreover, it is not present cumulative pollutant effects. However, it is a very toxic product both for humans and animals. The intoxication produced can be fatal mainly by the lack of an efficient antidote to revert the clinical state of the subject. Paraquat acts on the oxidative stress-induced mechanisms. Thus, there is the increased production of the free radicals associated with the depletion of antioxidant systems of the organism. Paraquat toxicity attacks kidneys, liver, muscles, and brain, but lungs are the target organs. Severe injuries are observed such as edema, hemorrhage, interstitial inflammation and pulmonary fibrosis, culminating in serious respiratory failure with death. Nowadays, the treatment of paraquat intoxication is based in decrease of the absorption and increases the excretion. Moreover, the use of antioxidants and antifibrotic agents has being studied. There is an increasing interest in studies about substances that can serve as antidote in the poisonings, once paraquat increases the morbidity and mortality.
\end{abstract}

Key words: Paraquat, herbicide, poisoning, pulmonary damage, clinical state, therapeutic.

\section{INTRODUÇÃO}

O controle ou eliminação de plantas invasoras tem sido uma meta da humanidade desde muito tempo. A situação ideal seria poder controlá-las ou eliminá-las, melhorando as espécies que fazem parte

\footnotetext{
'Programa de Pós-graduação em Bioquímica Toxicológica, Centro de Ciências Naturais e Exatas (CCNE), Universidade Federal de Santa Maria (UFSM), Santa Maria, RS, Brasil.

"Programa de Pós-graduação em Ciências Farmacêuticas, Centro de Ciências da Saúde (CCS), UFSM, Santa Maria, RS, Brasil.

IIICurso de Farmácia, CCS, UFSM, Santa Maria, RS, Brasil.

Iv Departamento de Tecnologia dos Alimentos, Centro do Ciências Rurais (CCR), UFSM, Santa Maria, RS, Brasil.

${ }^{\vee}$ Departamento de Análises Clínicas e Toxicológicas, CCS, UFSM, 97110-970, Santa Maria, RS, Brasil. E-mail: sgarpom@smail.ufsm.br. Autor para correspondência.
} 
do cultivo, sem alterar o equilíbrio ambiental. O aparecimento da agropecuária comercial e o decréscimo da mão-de-obra humana em tal setor trouxeram o rápido desenvolvimento de produtos químicos para o controle seletivo de ervas daninhas. Assim, o emprego de herbicidas tem eliminado numerosas intervenções de natureza mecânica que se aplicavam sobre os terrenos de cultivo. Atualmente, usam-se herbicidas tanto seletivos como não-seletivos, alguns altamente tóxicos e outros quase atóxicos (BUCK et al., 1981).

O paraquat (1-1'-dimetil-4-4'-bipiridilo), um herbicida de contato não-seletivo, é um sal de amônio bi-quaternário, usualmente sintetizado na forma de sal diclorídrico. Não existe em estado natural e sua síntese deve-se a Widel \& Russo, que publicaram seus trabalhos em 1882. No entanto, as propriedades herbicidas do produto só foram descobertas em 1955 (SERRA et al., 2003) e sua utilização iniciou-se a partir de 1962 (HONG et al., 2002). É amplamente utilizado em mais de 100 países (PETER et al., 1992) em culturas de fumo, algodão, arroz, café, cana-de-açúcar, feijão, maçã, soja, uva, dentre outras.

É um sólido incolor, cristalino e higroscópico. Sua fórmula molecular é $\mathrm{C}_{12} \mathrm{H}_{14} \mathrm{~N}_{2}$, com peso molecular de 186,25; na forma de dicloreto, $\mathrm{C}_{12} \mathrm{H}_{14} \mathrm{~N}_{2} \mathrm{Cl}_{2}$, apresenta peso molecular de 257,25 (CHAN et al., 1998). Não é volátil, explosivo ou inflamável em solução aquosa. É corrosivo para metais e estável em solução ácida ou neutra, mas hidrolisa-se facilmente em meio alcalino. Os seus sais são eletrólitos fortes que, em solução, dissociam-se em uma grande quantidade de íons positivos e negativos (SERRA et al., 2003). É solúvel em água, tem baixa solubilidade em álcoois e é insolúvel em hidrocarbonetos (CHAN et al., 1998). Geralmente é comercializado como Gramoxone ${ }^{\circledR}$, Gramocil $^{\circledR}$, Agroquat $^{\circledR}$, Gramuron $^{\circledR}$, Paraquat $^{\circledR}$, Paraquol ${ }^{\circledR}$ e também em misturas com outros princípios ativos, como o Secamato ${ }^{\circledR}$ (OGA et al., 2003).

O paraquat apresenta baixo custo, grande eficácia como herbicida e ausência de efeitos poluentes cumulativos para o solo (HONORE et al., 1994; SERRA et al., 2003; XARAU \& LAITA, 2000). Ao se depositar na superfície dos vegetais, sofre degradação fotoquímica através dos raios solares e ultravioleta, originando compostos menos tóxicos que o produto inicial (HONORE et al., 1994). Microorganismos do solo também podem degradá-lo (HAMADI et al., 2004) e, quando atinge o solo, é rápida e ativamente adsorvido pelos minerais argilosos (SERRA et al., 2003).

Em contrapartida, o paraquat é um produto muito tóxico, sendo capaz de causar intoxicações fatais tanto para humanos quanto para animais (MELCHIORRI et al., 1996). Ele tem sido usado para intoxicação intencional em cães (BEASLEY, 1999). Apresenta índice de mortalidade superior a 70\% em humanos (NAITO \& YAMASHITA, 1987), principalmente pela falta de um antídoto eficaz para reverter o quadro clínico (SERRA et al., 2003).

Pelo fato de ser considerado um herbicida não poluente ambientalmente, o paraquat é muitas vezes utilizado de forma indiscriminada, aumentando seu potencial toxicológico. Assim, o objetivo desta revisão bibliográfica é reunir dados relacionados à toxicologia veterinária do paraquat, chamando a atenção para a sua importância quanto à gravidade relacionada aos casos clínicos em animais intoxicados e, com isto, sugerir uma reavaliação no uso deste herbicida.

\section{Toxicocinética}

O paraquat pode ser absorvido em todo o trato gastrointestinal (FAO/WHO, 1978). Aproximadamente 20\% do paraquat ingerido oralmente é absorvido (BEASLEY, 1999). O paraquat não é absorvido em grande extensão pela pele íntegra e não há evidência de absorção significativa pelo spray misto (FAO/WHO, 1978). Não é absorvido por via respiratória, pois o paraquat é considerado não-volátil, uma vez que sua pressão de vapor ou volatibilidade é tão pequena que não é mensurável (FAO/WHO, 1978).

A maioria do paraquat é excretada inalterada na urina, embora possa ser metabolizado, e, aproximadamente $15 \%$ do paraquat absorvido é eliminado pelas fezes (BEASLEY, 1999). Porém, existem descrições de que a administração oral de paraquat na forma de dicloreto em ratos resulta em 94\% de excreção nas fezes e em 6\% na urina dentro de 48 horas (FAO/ WHO, 1978).

\section{Toxicodinâmica}

Nas plantas, o paraquat exerce sua atividade herbicida por interferir no sistema intracelular de transferência de elétrons, inibindo a redução de NADP a NADPH durante a fotossíntese, quando os radicais superóxido $\left(\mathrm{O}_{2}^{-}\right)$, oxigênio singlete $\left(\mathrm{O}_{2}^{*}\right)$, hidroxila $\left(\mathrm{OH}^{-}\right)$ e peróxido de hidrogênio $\left(\mathrm{H}_{2} \mathrm{O}_{2}\right)$ são formados nas plantas (RANJBAR et al., 2002). Este processo leva à destruição dos lipídios das membranas celulares pela polimerização de compostos lipídicos insaturados (SANDY et al., 1986).

Tem-se proposto que o mecanismo de dano tecidual deve-se ao aumento na formação de radicais livres e espécies reativas de oxigênio, entre eles o radical superóxido $\left(\mathrm{O}_{2}^{-}\right)$, o peróxido de hidrogênio $\left(\mathrm{H}_{2} \mathrm{O}_{2}\right)$ e o radical hidroxila $\left(\mathrm{OH}^{-}\right)$. Estas espécies são instáveis e reagem rapidamente com ácidos graxos, provocando 
lesão nas membranas, proteínas e DNA (PETER et al., 1992).

Inicialmente, o paraquat reage com uma substância doadora de elétrons, o NADPH, sofrendo uma redução por ação da enzima NADPH-citocromo $\mathrm{P}_{450}$ redutase, resultando na geração de um radical paraquat. Entretanto, sob condições aeróbicas, este elétron é transferido ao oxigênio, que se transforma em ânion superóxido. Como há suprimento de oxigênio no tecido pulmonar, o radical paraquat rapidamente se auto-oxida, produzindo radicais de ânion superóxido e regenerando o paraquat (FARRINGTON et al., 1973; SMITH \& HEATH, 1976). Na presença de suprimento suficiente de equivalentes reduzidos, repetidos ciclos de redução e reoxidação do herbicida podem ocorrer (PETER et al., 1992).

O ânion superóxido que se forma pode ser detoxificado pela ação da enzima superóxido dismutase (SOD) produzindo peróxido de hidrogênio. Este produto formado é removido através da enzima catalase. A SOD pode ser suprimida pela grande quantidade de superóxido que vai sendo produzida quando há doses tóxicas de paraquat. Dessa forma, os ânions superóxido sofrem uma reação de dismutação não-enzimática formando o oxigênio singlete, que ataca os lipídios insaturados das membranas celulares, dando origem aos hidroperóxidos lipídicos (BUS et al., 1975).

Radicais lipídicos livres, que se formam espontaneamente, reagem com lipídios poliinsaturados das membranas, formando mais radicais livres lipídicos. Isso leva, então, à lipoperoxidação devido à produção de radicais hidroxila, produzidos pelo peróxido de hidrogênio na presença de metais de transição na forma iônica, como ferro (BUS et al., 1975).

Como conseqüência, o balanço entre a geração de radicais de oxigênio, normalmente associada com o metabolismo celular, e sua dissipação pelos sistemas celulares de defesa - SOD, catalase, peroxidase, glutationa, vitamina E - é alterado, possibilitando que espécies reativas de oxigênio ataquem as biomoléculas (PETER et al., 1992).

O dano tecidual decorrente ocorre principalmente nos pulmões e é caracterizado pela necrose dose-dependente dos pneumócitos do tipo I e por congestão, edema e hemorragia pulmonares (REBELLO \& MASON, 1978; KELLY et al., 1978). Os pneumócitos do tipo II proliferam em resposta à perda de células do epitélio alveolar e progressiva fibrose intersticial ocorre após vários dias. Outros efeitos tóxicos do paraquat incluem necrose do miocárdio, necrose hepática periacinar, necrose dos túbulos renais e necrose adrenocortical (KELLY et al., 1978). Não existem valores estabelecidos de dose tóxica letal para animais. No entanto, a dose letal média $\left(\mathrm{DL}_{50}\right)$ via oral é de $35 \mathrm{mg} \mathrm{kg}^{-1}$ para gatos; $25-50 \mathrm{mg} \mathrm{kg}^{-1}$ para cães e suínos; 50-75mg kg-1 para ovinos e de 35-50mg kg-1 para bovinos (BEASLEY, 1999).

\section{Sinais clínicos}

Aparentemente, cães e bovinos têm sido intoxicados mais freqüentemente do que outras espécies de animais domésticos. Em cães, por exemplo, as intoxicações descritas ocorreram acidentalmente, por ingestão de grama tratada previamente com o herbicida (JOHNSON \& HUXTABLE, 1976), carcaça tratada, vômito de outro cão intoxicado e de forma não identificada em ambiente rural onde houve aplicação do produto (DARKE et al., 1977) ou intencionalmente (BISCHOFF et al., 1998; COPE, et al., 2004; SHULER et al., 2004). O rebanho pode acidentalmente ter acesso às áreas pulverizadas e, em muitas circunstâncias, como em áreas de silvicultura e em terrenos não cercados, é impossível manter o rebanho sempre longe da pastagem pulverizada (CALDERBANK et al., 1968). A intoxicação por água contaminada já foi descrita em ovinos (PHILBEY \& MORTON, 2001).

Após a exposição, os sinais clínicos da intoxicação podem levar de várias horas a três dias para terem início. Se grandes quantidades de paraquat são ingeridas, os sinais clínicos agudos podem ser observados incluindo excitação, ataxia, diarréia, angústia respiratória e convulsões (BEASLEY, 1999).

As superfícies que entram em contato com o paraquat podem ser irritadas e até ulceradas. Em cães, o começo dos sinais clínicos é tipicamente 1-3 dias após a exposição. Os sinais clínicos iniciais nestes animais incluem vômito e depressão, bem como desidratação. Os efeitos pulmonares iniciais são dosedependentes e os sinais respiratórios usualmente iniciam 2-7 dias após a exposição, incluindo taquipnéia, dispnéia, estertor úmido e cianose. A morte pode ocorrer dentro de 8 dias. Pneumomediastino, ocasionalmente, ocorre como um resultado da angústia respiratória. $\mathrm{O}$ prejuízo pulmonar progressivo pode continuar se desenvolvendo até um período de sete dias ou mais, porém, com 21 dias, é o dano mais acentuado observado em animais intoxicados pelo paraquat. Muitos animais morrem do edema pulmonar, enquanto outros sobrevivem somente até desenvolverem uma severa fibrose pulmonar em uma semana ou mais (BEASLEY, 1999).

A intoxicação por paraquat em bovinos pode ser notada 1-3 dias após a ingestão. Anorexia, depressão e diarréia podem ocorrer, e os sinais respiratórios são um tanto menos prevalentes do que em cães (BEASLEY, 1999). Em ovinos intoxicados, os 
sinais clínicos observados foram depressão, caminhar com cabeça abaixada e com andar incoordenado, perda de apetite, fraqueza, relutância em mover-se, orelhas caídas e pequeno interesse em seu redor. Com menor freqüência, observou-se membranas conjuntivais avermelhadas, diarréia aquosa amarela e desidratação (PHILBEY \& MORTON, 2001).

A intoxicação oral experimental por paraquat em perus produziu diarréia, alienação e anorexia com convulsões terminais. Em eqüinos, os estudos realizados com paraquat na pastagem mostraram a produção de efeitos tóxicos definidos. Dentre eles, notou-se uma seqüência de desenvolvimento de lesões na boca, iniciando com sinais de umidade ao redor dos lábios e leve flacidez da boca, evoluindo para copiosa secreção das glândulas labiais e inflamação dos lábios, língua e palato (CALDERBANK et al., 1968). Raramente, cavalos expostos desenvolveram hiperexcitabilidade, incoordenação e convulsões logo após a exposição. A congestão pulmonar severa e a fibrose ocorreram 3 a 5 dias e 7 a 10 dias, respectivamente, após a exposição (MURPHY, 1995).

A ingestão acidental, bem como maliciosa, de suínos têm resultado em toxicose. Os efeitos agudos envolvendo necrose e erosão das mucosas oral e gástrica são atribuídos ao solvente. Os efeitos mais clássicos, entretanto, ocorrem 7-10 dias após a ingestão e são caracterizados por congestão e edema pulmonar. As lesões pulmonares progridem para uma severa fibrose pulmonar. Os sinais clínicos iniciais incluem emese e diarréia, enquanto os estágios posteriores são caracterizados por disfunção respiratória. Uma vez que os sinais clínicos sugiram envolvimento pulmonar, medidas terapêuticas são usualmente inúteis (CARSON, 1992).

Diagnóstico

Com uma anamnese apropriada e a observação dos sinais clínicos e lesões, o diagnóstico pode ser feito. As alterações histológicas também são importantes no diagnóstico e deve ser feita uma análise da fonte de intoxicação suspeita com detecção de quantidades potencialmente tóxicas. Em muitos casos, por causa da rápida excreção, a urina pode ser analisada, mas seria de maior valor nos primeiros 2 dias ou logo após a exposição. Este teste pode confirmar a exposição antes do que necessariamente estabelecer o diagnóstico (BEASLEY, 1999).

De maneira rápida e fácil, o paraquat pode ser identificado na urina através do teste com solução alcalina de ditionito de sódio 1\%, em que a mudança de coloração da amostra para azul significa sua presença acima de $0,5 \mathrm{mg} \mathrm{L}^{-1}$ de urina. O teste é feito com os respectivos controles, positivo e negativo, e deve ser realizado nas primeiras 24 horas da intoxicação. $\mathrm{O}$ tom de azul poderá variar de acordo com a gravidade do caso e, por conseguinte, fornecer um prognóstico da sobrevivência (CALDAS, 2000).

Vários métodos têm sido utilizados na determinação de paraquat em matrizes aquosas, soro e urina. Eles incluem colorimetria com reação alcalina de ditionito (LIN et al., 1996), cromatografia em camada delgada (IKEBUCHI et al., 1988), cromatografia líquida de alta eficiência (BRUNETTO et al., 2003; ITO et al., 2005), cromatografia gasosa (KAWASE \& KANNO, 1984), eletroforese capilar (SANTILIO et al., 2005) e imunoensaios (BOWLES et al., 1992).

\section{Tratamento}

Em casos de intoxicação, o tratamento baseia-se essencialmente em três pontos: prevenção da absorção, rápida excreção do paraquat absorvido e modificação dos efeitos teciduais do absorvido e não excretado (SERRA et al., 2003).

A rápida remoção é o aspecto mais importante do tratamento. Para remoção, deve-se induzir vômitos, utilizando substâncias eméticas como xarope de ipeca ou, por estimulação mecânica, realizar lavagem gástrica e administrar laxantes (BOTELLA DE MAGLIA \& TARIN, 2000; SERRA et al., 2003). Um laxante salino (sulfato de magnésio ou sódio) ou um laxante osmótico (sorbitol) deve ser dado em todos os casos, quando os animais são apresentados depois de exposição recente (últimas 12 horas) (BEASLEY, 1999). As substâncias adsorventes como bentonita ou terra de Fuller possuem alta afinidade pelo paraquat, por esta razão, são os adsorventes preferidos para o herbicida (BOTELLA DE MAGLIA \& TARIN, 2000; SERRA et al., 2003). Quando estes agentes não estão imediatamente disponíveis, deve-se preferencialmente usar carvão ativado. A administração oral de terra argilosa pode servir como um absorvente de valor (BEASLEY, 1999).

Para a excreção do paraquat absorvido, medidas como a diurese forçada (BEASLEY, 1999; KUWAHARA et al., 1994, LHEUREUX et al., 1995), diálise peritonial (BEASLEY, 1999), hemodiálise, hemoperfusão e plasmaferese (KUWAHARA et al., 1994, LHEUREUX et al., 1995) podem ser adotadas.

Sem dúvida, o maior interesse no tratamento das intoxicações por paraquat tem se concentrado em medidas que impeçam ou minimizem as lesões celulares, principalmente aquelas provocadas por ele nos diversos órgãos atingidos, como nos pulmões (RIVERO GONZÁLES et al., 2001; SERRA et al., 2003). A ação tóxica do herbicida deve-se à ocorrência de uma reação 
cíclica de oxirredução com formação de radicais livres, especialmente nos pneumócitos do tipo I e II. Esta reação produz a depleção de diversas enzimas com papel fundamental para o organismo na ação antioxidante. Nesse sentido, tem se enfatizado a utilização de algumas substâncias antioxidantes. Agentes que têm se mostrado benéficos em intoxicações por paraquat experimentais incluem nacetil-cisteína (HONORE et al., 1994), ácido ascórbico (HONG et al., 2002), á-tocoferol (BLOCK, 1979), superóxido dismutase, ácidos graxos monoinsaturados como o ácido oléico (FRITZ et al., 1994), betabloqueadores (PATEL \& DAY, 1999), melatonina (MELCHIORRI et al., 1996), quelantes do ferro como desferroxamina e hidroxipiridin-4-ona (VAN ASBECK et al., 1989; VAN DER WAL et al., 1990; VAN DER WAL et al., 1992), metalotioneína (SATOH et al., 1992), litoespermato (YOKOZAWA et al., 1998), corticosteróides (LIN et al.,1996), talidomida, montelucaste e ácido acetil salicílico associados (CALDAS, 2004) e outras substâncias como o curcumin (VENKATESAN, 2000) e a 3-metil-1-fenil-2-pirazolin-5ona (SAIBARA et al., 2003).

Outras medidas gerais de suporte incluem a administração de fluidos e eletrólitos e o controle da dor (SITTIPUNT, 2005). A suplementação de oxigênio é contra-indicada devido à possibilidade de agravamento da injúria oxidativa pulmonar (BEASLEY, 1999).

A determinação e quantificação dos níveis de paraquat são fundamentais durante o tratamento e a recuperação. Isso porque o herbicida não sofre biotransformação significativa no organismo, e seus doseamentos no soro e na urina constituem dados importantes na avaliação do grau de exposição ao tóxico e do prognóstico (BRUNETTO et al., 2003).

A gasometria arterial seriada possui um bom valor preditivo na evolução e no prognóstico do quadro. Para tanto, sugere-se o uso do Índice Respiratório (IR) como um método simples para prever a probabilidade de sobrevivência do paciente intoxicado (PROUDFOOT et al., 1979; SUZUKI et al., 1989).

Medidas de prevenção

Vários países baniram ou restringiram severamente o uso do paraquat (ECOBICHON, 1991). Além disso, medidas como diminuir a concentração de paraquat e acrescentar substâncias odoríferas, corantes e eméticas nas preparações comerciais foram adotadas (XARAU \& LAITA, 2000). Nos EUA, por exemplo, sua utilização foi restrita somente ao pessoal treinado. Em muitos países, ainda há uso indiscriminado, permitindo que pessoas não treinadas manuseiem facilmente o paraquat na sua forma concentrada. Além disso, o fato de ele freqüentemente não ser armazenado no seu recipiente original contribui para intoxicações acidentais (ERICKSON et al., 1997). Para aves, o selênio na dieta funciona como um protetor contra a intoxicação aguda por paraquat (COMBS \& PETERSON, 1983; JULIAN \& BROWN, 1997).

\section{CONCLUSÃO}

Embora o paraquat seja ambientalmente correto, sua toxicidade coloca seu uso em questionamento. A intoxicação por paraquat, tanto em humanos como em animais, pode ser tomada como um exemplo de insegurança quanto à linha terapêutica a ser adotada.

Geralmente, as intoxicações agudas são fatais. Os sinais clínicos variam entre os animais, aparecendo desde horas até três dias após a exposição, e a depressão apresenta-se com maior freqüência. Para o diagnóstico laboratorial, a reação utilizando a solução alcalina de ditionito de sódio $1 \%$ na urina do animal suspeito de intoxicação, apesar de simples e rápida, é ainda uma identificação importante.

Conhecer totalmente o mecanismo de toxicidade e instituir novas e eficazes formas de tratamento para animais intoxicados, de forma acidental ou maliciosa, ainda é um desafio. Um caminho promissor parece ser a adoção de antioxidantes eficientes para evitar a principal causa de morte que é a fibrose pulmonar.

No entanto, a eficácia das terapias mais recentemente desenvolvidas continua sob avaliação, sendo necessários mais estudos clínico-toxicológicos sobre o assunto.

Portanto, o manejo e a utilização deste herbicida devem ser reavaliados, procurando-se alternativas de uso cauteloso, responsável e profissional, evitando-se assim, o que ainda não tem solução, a reversão do quadro toxicológico.

\section{REFERÊNCIAS}

BEASLEY, V. Organic compounds that affect the lungs. In: Veterinary toxicology. Ithaca: International Veterinary Information Service, 1999. Capturado em 2 mar. 2006. Online. Disponível na Internet: http://www.ivis.org/ advances/Beasley/Cpt15b/chapter_frm.asp?LA=1\#.

BISCHOFF, K. et al. Malicious paraquat poisoning in Oklahoma dogs. Veterinary and Human Toxicology, v.40, n.3, p.151153, 1998.

BLOCK, E.R. Potentiation of acute paraquat toxicity by vitamin E deficiency. Lung, v.156, n.3, p.195-203, 1979.

Ciência Rural, v.37, n.5, set-out, 2007. 
BOTELLA DE MAGLIA, J.; TARIN, J.E.B. Intoxicación por paraquat. Estudio de 29 casos y evaluación del tratamiento con la “pauta caribeña”. Medicina Clínica, v.115, p.530-533, 2000.

BOWLES, M.R. et al. Quantitation of paraquat in biological samples by radioimmunoassay using a monoclonal antibody. Fundamental and Applied Toxicology: Official Journal of the Society of Toxicology, v.19, n.3, p.375-379, 1992.

BRUNETTO, M.R. et al. Determination of paraquat in human blood plasma using reversed-phase ion-pair high-performance liquid chromatography with direct sample injection. Talanta, v.59, p.913-921, 2003

BUCK, W.B. et al. Toxicología veterinaria clínica y diagnóstica. Zaragoza: Acribia, 1981. 475p.

BUS, J.S. et al. Lipid peroxidation: a possible mechanism for paraquat toxicity. Research Communications in Chemica Pathology and Pharmacology, v.11, n.1, p.31-38, 1975.

CALDAS, L.Q.A. Intoxicações exógenas agudas por carbamatos, organofosforados, compostos bipiridílicos e piretróides. Niterói: Centro de Controle de Intoxicações de Niterói, 2000. 40p.

CALDAS, L.Q.A. et al. Tratamento da intoxicação experimental por paraquat com talidomida, montelucaste e ácido acetil salicílico. Revista Brasileira de Toxicologia, v.17, n.1, p.11-15, 2004.

CALDERBANK, A. et al. Grazing trials on paraquat-treated pasture. Journal of Tropical Agriculture and Food Science, v.19, p.246-250, 1968

CARSON, T.L. Toxic minerals, chemicals, plants and gases. In: LEMAN, A.D. et al. Diseases of swine. 7.ed. Ames: Iowa State University, 1992. Cap.64, p.777-790.

CHAN, B.S. et al. The renal excretory mechanisms and the role of organic cations in modulating the renal handling of paraquat. Pharmacology \& Therapeutics, v.79, n.3, p.193203, 1998.

COMBS JUNIOR, G.F.; PETERSON, F.J. Protection against acute paraquat toxicity by dietary selenium in the chick. Journal of Nutrition, v.113, n.3, p.538-545, 1983.

COPE, R.B. et al. Fatal paraquat poisoning in seven Portland, Oregon, dogs. Veterinary and Human Toxicology, v.46, n.5, p.258-264, 2004.

DARKE, P.G.G. et al. Acute respiratory distress in the dog associated with paraquat poisoning. Veterinary Record, v.100, p.275-277, 1977.

ECOBICHON, D.J. Toxic effects of pesticides. In: AMDUR, M.O. et al. Casarett and Doull's toxicology: the basic science of poisons. 4.ed. New York: Mc Graw Hill, 1991 Cap.22, p.763-810.

ERICKSON, T. et al. A case of paraquat poisoning and subsequent fatality presenting to an emergency department. Journal of Emergency Medicine, v.15, n.5, p.649-652, 1997.
FAO/WHO. Data sheets on pesticides. n.1, rev.1, 1978. Capturado em 13 fev. 2000. Online. Disponível na Internet: http://www.inchem.org/documents/pds/pds/pest4_e.htm.

FARRINGTON, J.A. et al. Bipyridylium quaternary salts and related compounds. V. Pulse radiolysis studies of the reaction of paraquat radical with oxygen. Implications for the mode of action of bipyridyl herbicides. Biochimica et Biophysica Acta, v.314, n.3, p.372-381, 1973

FRITZ, K.L. et al. Acute intramuscular injection of oils or the oleic acid component protects mice against paraquat lethality. Journal of Nutrition, v.124, n.3, p.425-429, 1994.

HAMADI, N.K. et al. Adsorption os paraquat dichloride from aqueous solution by activated carbon derived from used tires. Journal of Hazardous Materials, v.112, p.133-141, 2004.

HONG, S.Y. et al. Effect of vitamin C on plasma total antioxidant status in patients with paraquat intoxication. Toxicology Letters, v.126, p.51-59, 2002.

HONORE, P. et al. Paraquat poisoning. 'State of the art'. Acta Clinica Belgica, v.49, n.5, p.220-228, 1994

IKEBUCHI, J. et al. A rapid and sensitive method for the determination of paraquat in plasma and urine by thin-layer chromatography with flame ionization detection. Journal of Analytical Toxicology, v.12, n.2, p.80-83, 1988.

ITO, M. et al. Rapid analysis method for paraquat and diquat in the serum using ion-pair high-performance liquid chromatography. Biological \& Pharmaceutical Bulletin, v.28, n.4, p.725-728, 2005.

JOHNSON, R.P.; HUXTABLE, C.R. Paraquat poisoning in a dog and cat. Veterinary Record, v.98, p.189-191, 1976

JULIAN, R.J; BROWN, T.P. Poisons and toxins / Other toxins and poisons. In: CALNEK, B.W. et al. Diseases of poultry. 10.ed. Ames: Iowa State University, 1997. Cap.36, p.979-1005.

KAWASE, S.; KANNO, S. Determination of the herbicides paraquat and diquat in blood and urine by gas chromatography. Journal of Chromatography A, v.283, p.231-240, 1984.

KELLY, D.F. et al. Pathology of acute respiratory distress in the dog associated with paraquat poisoning. Journal of Comparative Pathology, v.88, p.275-294, 1978.

KUWAHARA, M. et al. Hemoperfusion and plasma exchange therapy in patients with paraquat poisoning. Hinyokika Kiyo, v.40, n.5, p.439-442, 1994.

LHEUREUX, P. et al. Survival in a case of massive paraquat ingestion. Chest, v.107, n.1, p.285-289, 1995.

LIN, J. et al. Pulse therapy with cyclophosphamide and methylprednisolone in patients with moderate to severe paraquat poisoning: a preliminary report. Thorax, v.51, p.661-663, 1996.

MELCHIORRI, D. et al. Paraquat toxicity and oxidative damage. Biochemical Pharmacology, v.51, p.1095-1099, 1996.

MURPHY, M.J. Toxicology. In: KOBLUK, C.N. et al. The horse: diseases and clinical management. Philadelphia: Sauders, 1995. 2v. Cap.50, p.1165-1174. 
NAITO, H.; YAMASHITA, M. Epidemiology of paraquat in Japan and new safe formulation of paraquat. Human Toxicology, v.6, n.1, p.87-88, 1987.

OGA, S. et al. Praguicidas. In: OGA, S. Fundamentos de toxicologia. 2.ed. São Paulo: Atheneu, 2003. Cap.5.3, p.467458 .

PATEL, M.; DAY, B.J. Metalloporphyrin class of therapeutic catalytic antioxidants. Trends in Pharmacological Sciences, v.20, n.9, p.359-364, 1999.

PETER, B. et al. Role of lipid peroxidation and DNA damage in paraquat toxicity and the interaction of paraquat with ionizing radiation. Biochemical Pharmacology, v.43, n.4, p.705715, 1992.

PHILBEY, A.W.; MORTON, A.G. Paraquat poisoning in sheep from contaminated water. Australian Veterinary Journal, v.79, n.12, p.842-843, 2001.

PROUDFOOT, A.T. et al. Paraquat poisoning: significance of plasma-paraquat concentrations. Lancet, v.314, n.8138, p.330-332, 1979.

RANJBAR, A. et al. Induction of oxidative stress in paraquat formulating workers. Toxicology Letters, v.131, p.191-194, 2002.

REBELLO, G.; MASON, J.K. Pulmonary histological appearances in fatal paraquat poisoning. Histhopathology, v.31, p.647-649, 1978.

RIVERO GONZÁLES, A. et al. Intoxicación por paraquat: presentación de dos casos y revisión de la literatura. Anales de Medicina Interna, v.18, n.4, p.208-210, 2001.

SAIBARA, T. et al. Protective effect of 3-methyl-1-phenyl2-pyrazolin-5-one, a free radical scavenger, on acute toxicity of paraquat in mice. Toxicology Letters, v.143, p.51-54, 2003.

SANDY, M.S. et al. Role of redox cycling and lipid peroxidation in dipyridyl herbicide cytotoxicity. Studies with a compromised isolated hepatocyte model system. Biochemical Pharmacology, v.35, n.18, p.3095-3101, 1986.

SANTILIO, A. et al. Analysis of bipyridylium herbicides by capillary zone electrophoresis. Journal of Capillary Electrophoresis, v.8, n.1-2, p.1-5, 2005.
SATOH, M. et al. Effect of preinduction of metallothionein on paraquat toxicity in mice. Archives of Toxicology, v.66, n.2, p.145-148, 1992.

SERRA, A. et al. Intoxicação por paraquat. Acta Médica Portuguesa, v.16, p.25-32, 2003.

SHULER, C.M. et al. Retrospective case series of suspected intentional paraquat poisonings: diagnostic findings and risk factors for death. Veterinary and Human Toxicology, v.46, n.6, p.313-314, 2004.

SITTIPUNT, C. Paraquat poisoning. Respiratory Care, v.50, n.3, p.383-385, 2005.

SMITH, P.; HEATH, D. Paraquat. CRC Critical Reviews in Toxicology, v.4, n.4, p.411-445, 1976.

SUZUKI, K. et al. A new method for predicting the outcome and survival period in paraquat poisoning. Human \& Experimental Toxicology, v.8, n.1, p.33-38, 1989.

VAN ASBECK, B.S. et al. Continuous intravenous infusion of deferoxamine reduces mortality by paraquat in vitamin Edeficient rats. American Review of Respiratory Disease, v.139, n.3, p.769-773, 1989.

VAN DER WAL, N.A.A. et al. Mechanism of protection of alveolar type II cells against paraquat-induced cytotoxicity by deferoxamine. Biochemical Pharmacology, v.39, n.11, p.1665-1671, 1990.

VAN DER WAL, N.A. et al. Effect of iron chelators on paraquat toxicity in rats and alveolar type II cells. American Review of Respiratory Disease, v.145, n.1, p.180-186, 1992.

VENKATESAN, N. Pulmonary protective effects of curcumin against paraquat toxicity. Pharmacology Letters, v.66, n.2, p.21-28, 2000.

XARAU, S.N.; LAITA, A.D. Intoxicación por paraquat: um puzzle al que le faltan piezas. Medicina Clínica, v.115, p.546548, 2000.

YOKOZAWA, T. et al. Confirmation that magnesium lithospermate B ameliorates paraquat-induced injury in cultures renal epithelial cells. Nephron, v.79, n.3, p.373-374, 1998. 